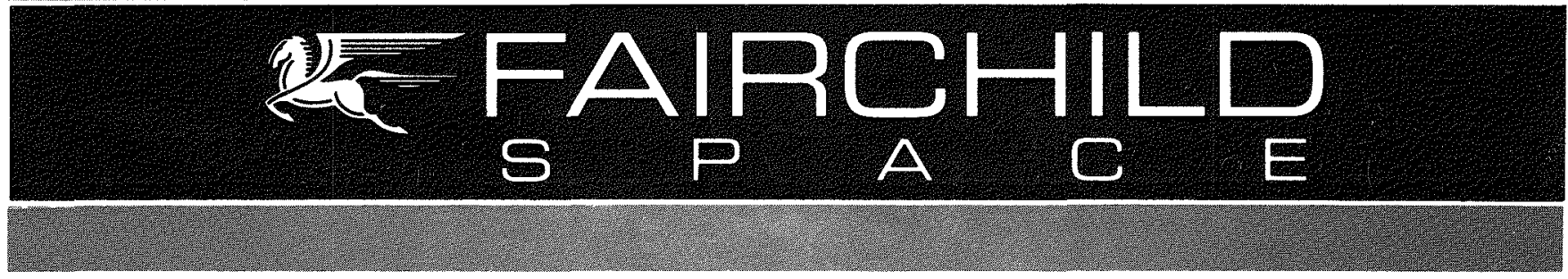

$\Gamma$

\#

IECEC\#910472

$a+$

\title{
CLOSED-FORM SOLUTION FOR THE EFFECT OF FUEL DECAY AND THERMOELECTRIC DEGRADATION ON OUTPUT OF SIGe RTGS
}

\author{
Alfred Schock \\ Fairchild Space Company \\ 20301 Century Blvd. \\ Germantown, MD 20874 \\ (301) 428-6272
}

For presentation at the

26th Intersociety Energy Conversion Engineering Conference

Boston, Massachusetts

August 4-9, 1991 


\section{DISCLAIMER}

This report was prepared as an account of work sponsored by an agency of the United States Government. Neither the United States Government nor any agency Thereof, nor any of their employees, makes any warranty, express or implied, or assumes any legal liability or responsibility for the accuracy, completeness, or usefulness of any information, apparatus, product, or process disclosed, or represents that its use would not infringe privately owned rights. Reference herein to any specific commercial product, process, or service by trade name, trademark, manufacturer, or otherwise does not necessarily constitute or imply its endorsement, recommendation, or favoring by the United States Government or any agency thereof. The views and opinions of authors expressed herein do not necessarily state or reflect those of the United States Government or any agency thereof. 


\section{DISCLAIMER}

Portions of this document may be illegible in electronic image products. Images are produced from the best available original document. 


\title{
CLOSED-FORM SOLUTION FOR THE EFFECT OF FUEL DECAY AND THERMOELECTRIC DEGRADATION ON OUTPUT OF SIGE RTGS
}

\author{
Altred Schock \\ Fairchild Space \\ 20301 Century Blvd. \\ Germantown, Maryland 20874
}

(301) 428-6272

\begin{abstract}
The paper derives a closed-form solution for the long-term effect of fuel decay and thermoelectric degradation on the performance of Radioisotope Thermoelectric Generators employing silicongermanium converter elements. RTGs of this type were used to power the recent Galileo and Ulysses space exploration missions, and are slated for use on the upcoming CRAF and Cassini missions. The method described applies not only to uniform-temperature RTGs, but also to RTGs with significant axial and circumferential variations in the couples' cold-junction temperatures and voltages (due to unsymmetrically obstructed heat rejection paths). This is important for the mutually blocking RTGs on the CRAF and Cassini spacecraft, and even more so for the reflector-blocked Solar Probe RTGs. The method for predicting RTG degradation that is derived in this paper is based on both analytical and experimental data. It accounts for the effect of diminishing hot-junction temperatures on thermoelectric degradation rates. The method leads to an integral equation, for which the author was able to derive a closed-form solution. The solution was successfully validated by comparison with long-term test data. It enables the RTG designer to predict the power output profile throughout the mission, to ensure that it satisfies the mission's power demand profile.
\end{abstract}

\section{INTRODUCTION}

Designers of spacecraft powered by Radioisotope Thermoelectric Generators (RTGs) need to know not only their beginning-of-mission (BOM) power but also how their power output diminishes during the mission as the result of fuel decay and of thermoelectric degradation. A general method of predicting the power loss for various BOM fuel loadings and thermal environments is needed for both unobstructed and obstructed RTGs.

This paper describes an analytical method of making such predictions for RTGs employing SiGe unicouples, like those flown on the LES-8/9, Voyager, Galileo, and Ulysses missions, and slated for use on the upcoming CRAF and Cassini missions. The analysis is based on a method of predicting the RTG's BOM power, and applying corrections for the effects of isotope decay and of thermoelectric degradation.
The BOM power prediction is based on novel methods developed by Fairchild for the coupled thermal, thermoelectric, and electrical analysis of RTGs that were described in other reports [1, 2]. Their novelty includes the ability to account for the RTG's axial and circumferential variation of hot-and cold-junction temperatures and the resultant variation of the couples' voltages and currents in the series-parallel network. This capability is particularly important for RTGs with unsymmetrically obstructed heat rejection paths, such as the CRAF/Cassini RTGs with mutual blockage between neighboring RTGs as illustrated in Figure 1.

Figure 1. CRAF/CASSINI Spacecraft, Initial Design

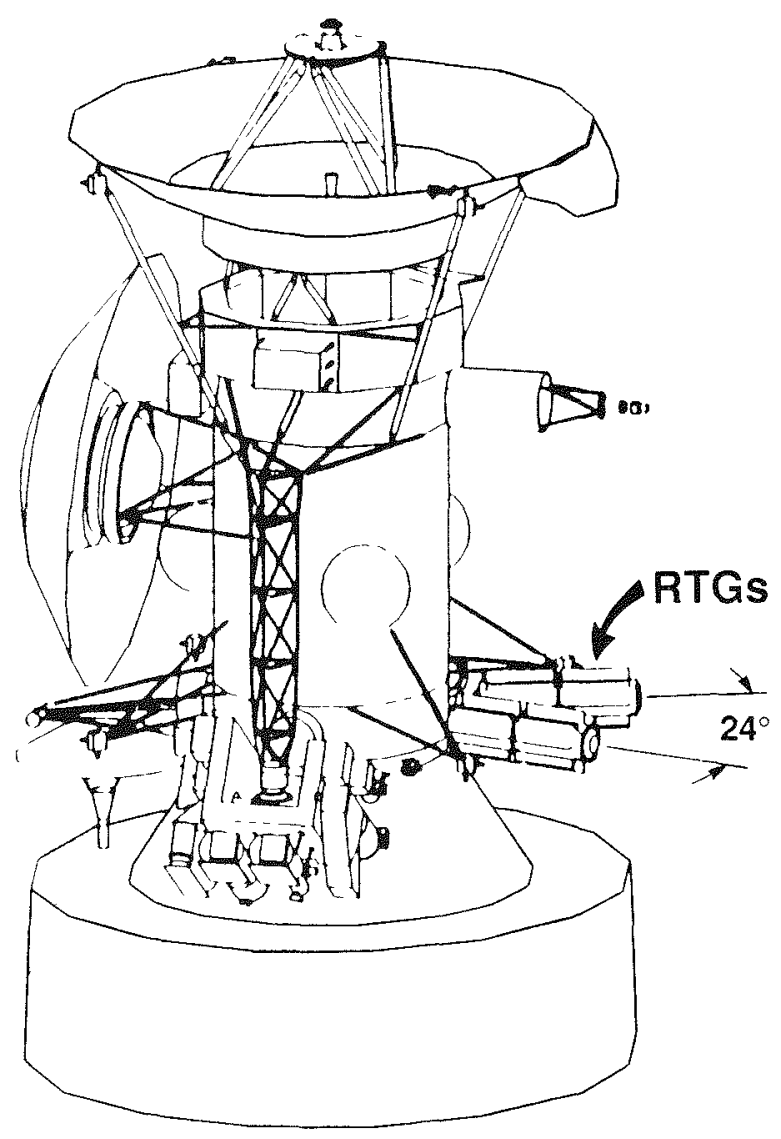




\section{INITIAL PERFORMANCE}

The coupled thermal and electrical analysis described in [1, 2] can be used to predict the BOM electrical power and mean and maximum hot-junction temperatures of the RTG as a function of fuel loading or BOM thermal power (for a specified thermal environment). When the results of the detailed analyses were plotted against thermal power, it was found that over a broad range of practical interest the undegraded output power $\mathbf{P}_{\mathbf{u}}$ and the hot-junction temperature $\mathrm{T}$ of both unobstructed and obstructed RTGs were linear functions of the RTG's thermal power $Q$ :

$$
\begin{aligned}
\mathbf{P}_{\mathrm{U}} & =\mathbf{P}^{*}+\eta^{*} \mathbf{Q}, \\
\mathbf{T} & =\mathbf{T}_{\mathbf{O}}+\alpha \mathbf{Q},
\end{aligned}
$$

where the coefficients $\mathbf{P}^{*}, \eta^{\star}, \mathrm{T}_{\mathbf{0}}$, and $\alpha$ are functions of the RTG's thermal environment. For example, for a GPHS-RTG mounted on the CRAF/Cassini spacecraft and separated from its neighbor by an angle of 35 degrees as shown in Figure 1, least-square analysis of computed results over the thermal-power range $4050 \leq$ $Q \leq 4500$ watts $(t)$ yielded

$$
\begin{aligned}
& P^{\star}=-218.0 \mathrm{w}, \\
& \eta^{\star}=0.1188, \\
& T_{0}=582.6^{\circ} \mathrm{K}, \text { and } \\
& \alpha=0.1565^{\circ} \mathrm{K} / \mathrm{w} .
\end{aligned}
$$

Note that the coefficient $\eta^{*}$ is a differential efficiency, which is the sum of the actual efficiency $\eta$ and a term that reflects the fact that increasing $Q$ increases the temperature drop $\Delta \mathrm{T}$ between hot- and cold-junctions, which in turn increases the efficiency:

$$
\eta^{*}=\frac{d P}{d Q}=\frac{d(Q \eta)}{d Q}=\eta+Q\left(\frac{\partial \eta}{\partial(\Delta T)}\right)\left(\frac{\partial(\Delta T)}{\partial Q}\right)
$$

Typically, the efficiency $\eta$ has a value of $7 \%$, and the $5 \%$ difference between $\eta$ and the differential efficiency $\eta^{\star}$ represents the last term of Eq. 3 .

\section{EFFECT OF FUEL DECAY}

The effect of fuel decay on the RTG's thermal power $Q$ at mission time $t$ is given by

$$
Q=Q_{l} \exp (-\lambda t)
$$

where $Q_{1}$ is the $B O M$ value of $Q$, and $\lambda$ is the $P u-238$ decay constant, which is related to the isotope's half-life $\tau$ ( 87.74 years) by

$$
\lambda=(\ln 2) / \tau
$$

Inserting Eqs. 4 and 5 into 1, we obtain an expression for the effect of fuel decay alone (i.e., without thermoelectric degradation) on the RTG's power output:

$$
\frac{P_{u}}{P_{1}}=1-\frac{1-e^{-\lambda t}}{\left(P^{*} / \eta^{*} Q_{1}\right)+1}
$$

where $P_{1}$ is the RTG's BOM power output.

\section{EFFECT OF THERMOELECTRIC DEGRADATION}

Predicting the thermoelectric performance degradation with time is generally a complex problem since many different degradation mechanisms are at work. A detailed method for making such predictions is the DEGRA code developed by $V$. Raag [3], but that code does not account for the detailed effects of mutual obstruction between neighboring RTGs. In the same period, A. Mowery [4] performed statistical analyses of the results of various tests on thermoelectric converters that were similar to the converters in the GPHS-RTGs, but which were electrically heated and which operated at constant thermal powers and constant temperatures. These statistical analyses showed that the measured power output of a constant-temperature converter is a linear function of the square root of time $t$, and that the coefficient of $\sqrt{t}$ is an Arrhenius function of the absolute hot-junction temperature $\mathrm{T}$. In the range of practical interest, Mowery found that the measured effect of thermoelectric degradation could be quite accurately correlated by a simple empirical equation equivalent to

$$
\begin{aligned}
& P / P_{u}=1-\left\{\exp \left[-T^{*} / T\right]\left(t / t^{*}\right)\right\}^{1 / 2} \\
& \text { where } T^{*}=30,960^{\circ} \mathrm{K} \text { and } t^{*}=0.648 \mathrm{sec}
\end{aligned}
$$

This empirical expression was found to give excellent agreement with the experimental data from the electrically heated tests at various constant thermal powers and temperatures. However, Mowery's equations cannot be directly applied to predict the performance degradation of RTGs, because an RTG operates at a diminishing thermal power and temperature, and therefore at diminishing degradation rates. To account for that effect, the present author proposed the following simple modification of Eq. 7:

$$
P / P_{u}=1-\left\{\int_{0}^{t} \exp \left[-T^{*} / T\left(t^{\prime}\right)\right] d t^{\prime} / t^{*}\right\}^{1 / 2}
$$

where $T\left(t^{\prime}\right)$ is the hot-junction temperature at time $t^{\prime}$. Note that for the special case of constant $T$, the modified equation reduces to Mowery's validated Eq. 7 . For a variable $\mathrm{T}$, the validity of $\mathrm{Eq} .8$ was subsequently confirmed by successfully predicting the results of longterm tests at diminishing temperatures. 
The time-varying temperature $T\left(t^{\prime}\right)$ in Eq. 8 is obtained by inserting Eq. 4 into 2, yieiding

$$
T\left(t^{\prime}\right)=T_{o}+\alpha Q_{1} \exp \left(-\lambda t^{\prime}\right) \text {. }
$$

Inserting Eq. 9 into 8, we obtain the expression

$$
\frac{P}{P_{u}}=1-\left\{\int_{0}^{t} \exp \left[-\frac{T^{*}}{T_{o}+\alpha Q_{1} \exp \left(-\lambda t^{\prime}\right)}\right] d t^{\prime} / t^{*}\right\}^{1 / 2}
$$

for the effect of thermoelectric degradation on power. At first glance Eq. 10 looks unintegrable, but by defining the dimensionless variable

$$
x \equiv T^{*} /\left[T_{o}+\alpha Q_{1} \exp \left(-\lambda i^{\prime}\right)\right]
$$

it can be reduced to

$$
\begin{gathered}
\frac{P}{P_{u}}=1-\left\{\frac{1}{\lambda t^{*}} \int_{x_{1}}^{x_{2}} \frac{\exp (-x) d x}{x\left[1-\left(T_{o} / T^{*}\right) x\right]}\right\}^{1 / 2}, \\
\text { where } x_{1} \equiv T^{*} /\left(T_{o}+\alpha Q_{1}\right) \\
\text { and } x_{2} \equiv T^{*} /\left(T_{o}+\alpha Q_{1} e^{-\lambda t}\right)
\end{gathered}
$$

Equation 12 can be partitioned into

$$
\begin{aligned}
\frac{P}{P_{u}}= & =\left\{\frac{1}{\left(\lambda t^{*}\right)} \Gamma \int_{x_{1}}^{x_{2}} \frac{e^{-x} d x}{x}+\right. \\
& \left.+\int_{x_{t}}^{x_{2}} \frac{e^{-x} d x}{\left(T^{*}\left(T_{o}\right)-x\right.}\right\}^{1 / 2}
\end{aligned}
$$

for which exact solutions in terms of exponential integrals are readily obtained. However, when we consider the magnitude of the $x$-values of interest, an almost exact but much simpler solution is available. Let us consider a typical problem with a BOM thermal power $\mathrm{Q}_{1}$ of 4410 watts and a mission life $t$ of 12 years. Thus, from Eqs. 13 and 14

$$
\begin{aligned}
& x_{1}=24.325, \\
& x_{2}=25.590 .
\end{aligned}
$$

For that range of $x$-values, the denominator in the integrand of Eq. 12 varies only from 13.18 to 13.26 , a range of only $\pm 0.3 \%$. Therefore, that denominator can be quite accurately approximated by its initial value, so that $\mathrm{Eq} .12$ reduces to

$$
\frac{P}{P_{u}}=1-\int_{x_{1}}^{x_{2}} e^{-x} d x
$$

Integration of Equation 16 gives

$$
\frac{P}{P_{u}}=1-\left\{\frac{\exp \left(-x_{1}\right)-\exp \left(-x_{2}\right)}{\left(\lambda t^{*}\right) x_{1}\left[1-\left(T_{o} / T^{*}\right) x_{1}\right]}\right\}^{1 / 2},
$$

which can also be expressed in terms of temperatures

$$
\begin{aligned}
& \frac{P}{P_{u}}=1-\left\{\frac{\exp \left(-T^{*} / T_{1}\right)-\exp \left(-T^{*} / T\right)}{\left(\lambda t^{*}\right)\left(T^{*} / \bar{T}\right)\left[1-\left(T_{o} / \bar{T}\right)\right]}\right\}, \\
& \text { where } T_{I}=T_{o}+\alpha Q_{1}, \\
& \text { and } T=T_{o}+\alpha Q_{1} e^{-\lambda t} .
\end{aligned}
$$

The above solution for the effect thermoelectric degradation accounts for diminishing temperatures and degradation rates with time.

To complete the analysis, we combine the effect of fuel decay with the effect of thermoelectric degradation

$$
\frac{P}{P_{1}}=\left(\frac{P_{u}}{P_{I}}\right)\left(\frac{P}{P_{u}}\right)
$$

Combining Eqs. 1, 4, and 18, we obtain the closed-form solution

$$
\begin{aligned}
P= & \left(P^{*}+\eta^{*} Q_{1} e^{-\lambda t}\right) \times \\
& \left\{1-\left\lceil\frac{\exp \left(-T^{*} / T_{1}\right)-\exp \left(-T^{*} / T\right)}{\left(\lambda t^{*}\right)\left(T^{*} / T_{I}\right)\left[1-\left(T_{o} / T_{I}\right)\right]}\right\rceil^{1 / 2}\right\}
\end{aligned}
$$

for the combined effect of fuel decay and thermoelectric degradation.

\section{VALIDATION OF DEGRADATION MODEL}

The above equations were applied to the $Q-1$ RTG, which was the qualification unit for the GPHSRTGs used on the Galileo mission. The computed results for a 12-year period are shown in Figure 2. The curve labeled $Q / Q_{1}$ shows the thermal power decrease due to isotope decay (Eq. 4), and the curve labeled $T$ shows the resultant decrease in average hot-junction temperature (Eq. 9). The curve labeled $P_{u} / P_{1}$ shows the power loss due to fuel decay for undegraded thermoelectric performance (Eq. 6), and the curve labeled $P / P_{u}$ shows the power loss due to thermoelectric degradation (Eq. 18). The combined effect of fuel decay and thermoelectric degradation is shown by the curve labeled $P / P_{1}$ (Eq. 21). As can be seen, the combined effect for this case is predicted to result in a $23.6 \%$ power loss in 12 years. 
Figure 2. Effect of Fuel Decay and Thermoelectric Degradation on Performance of Galileo

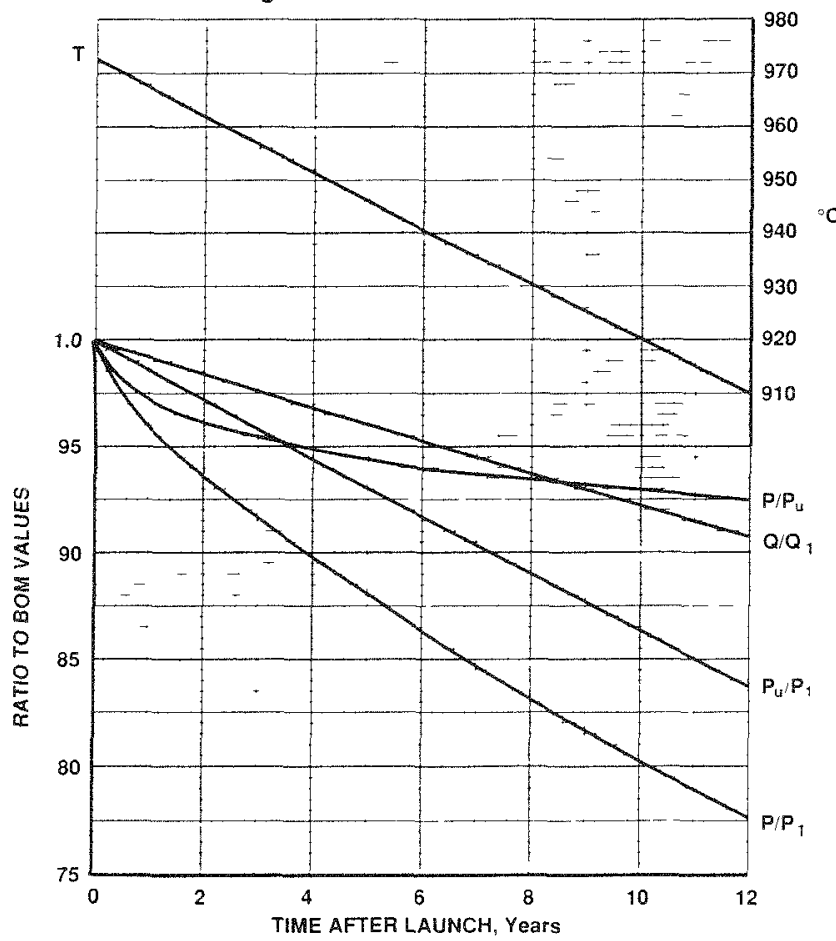

Figure 3 shows a comparison of these predictions with the measured results of a five-year test of the $Q-1$ RTG at diminishing temperatures. As can be seen, for the range of times and temperatures tested, the rather simple prediction model used showed surprisingly good agreement with the experimental results.

\section{APPLICATION OF PREDICTIVE ANALYSIS TO SPECIFIC MISSION}

The analytical methodology described above was used to predict the long-term power degradation for the original CRAF/Cassini design, in which two RTGs were mounted on each spacecraft (See Figure 1.) Parametric analytical results for two RTGs with a 35 degree separation angle are shown in Figure 4. For various fuel loadings, the solid curves show the variation of RTG power with time. For each solid curve, the figure shows the thermal power (in each of the 18 heat source modules) and the corresponding plutonium enrichment (for the same fuel density as that used in the Galileo RTGs). The numbers listed are for two points in time: at the chemical separation of the plutonium from the irradiated neptunium-237 targets (BOL), and three years later at launch (BOM). Thus, the figure can be employed as a useful design tool by the CRAF and Cassini mission planners.

For each solid curve in Figure 4, its intersections with the dashed curves indicate the decrease of the RTG's average hot-junction temperature with time for that fuel loading. (Each constant-temperature curve was obtained by inserting $T$ into $E q .20$, solving for $Q_{1}$ at each mission time, and using that value of $Q_{1}$ in Eq. 22 to compute the corresponding output power $P$.)
Figure 3. Comparison of Predicted and Measured Power History of Q-1 RTG

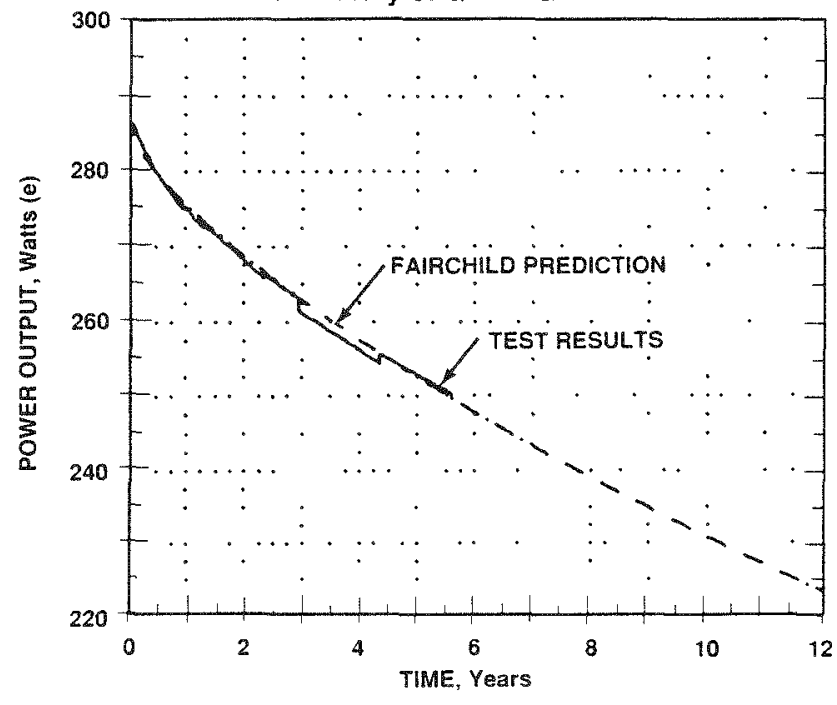

The bold solid curve in the figure represents the fuel loading in the Galileo RTGs, and the bold dashed curve represents the $1000^{\circ} \mathrm{C}$ hot-junction limit adopted in previous missions. Note that this limit is exceeded by the BOM temperatures at higher fuel loadings. But modest excesses may be allowed, since the curves displayed in Figure 4 already include proper allowances for increased degradation at the higher temperatures, based on results of (accelerated) tests conducted above $1000^{\circ} \mathrm{C}$.

Figure 4. Effect of Fuel Loading on RTGs Power and Temperature Histories

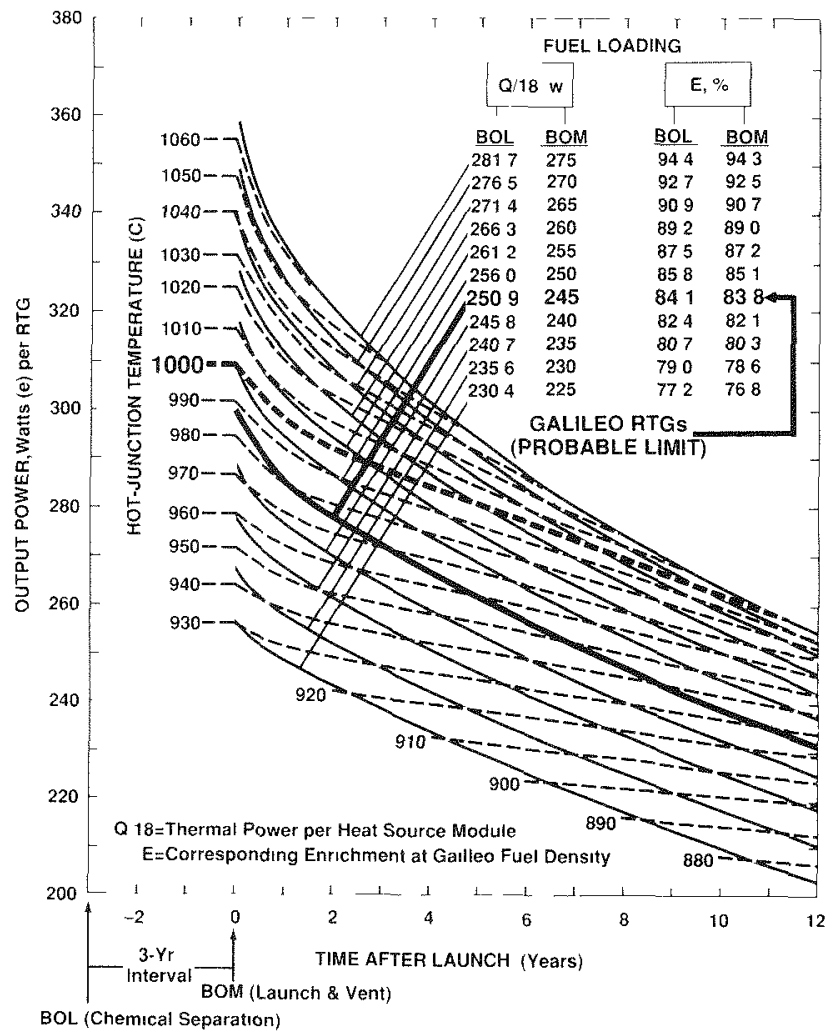


Figure 4 shows that increasing the BOM thermal power (by increasing the fuel loading) leads to a substantial increase of the RTG's electrical power at the beginning of mission, but that this benefit dimınishes towards the end of mission, particularly for high fuel loadings and long mission times. This comes about because higher thermal powers result in higher temperatures and therefore higher degradation rates. In fact, at unrealistically high hot-junction temperatures increasing the thermal power can actually decrease the EOM electrical power.

Figure 5. Mounting of Three RTGs on Mariner Mark-2 Spacecrait

(34० Separation Between Neighbors)

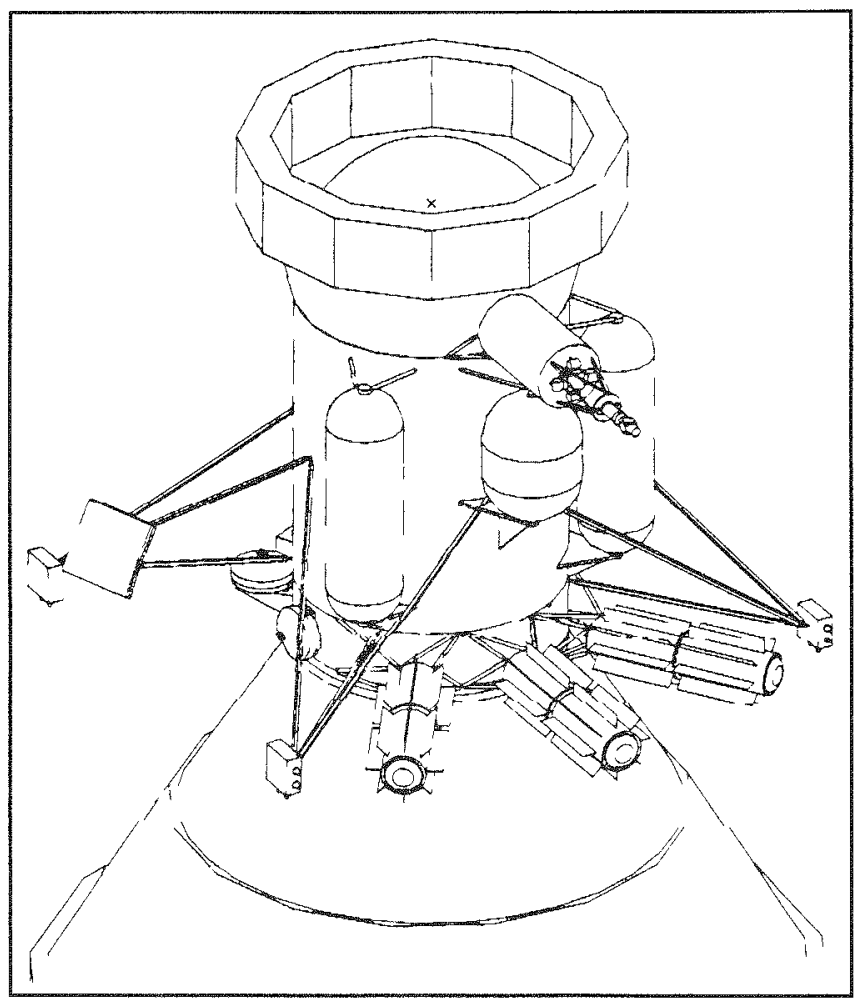

Subsequently, the CRAF and Cassini designs were modified to provide three instead of two RTGs per spacecraft. (See Figure 5.) These were subjected to the same prediction analysis, taking account of the greater blockage (and consequent higher temperatures and degradation rate of the middle RTG. The analytical predictions for a range of fuel loadings are shown in Figure 6.

Figure 6 shows the power output histories of three standard GPHS-RTGs for various fuel enrichments at the time of chemical separation, corresponding to thermal powers ranging from 225 to 255 watts (per heat source module) at launch (three years later). The curves shown extend for 12 years, covering both the CRAF and Cassini mission durations.

This and other applications to which the Fairchild methodology has been applied demonstrate its great utility.
Figure 6. Effect of BOM Thermal Power on Output of Three Std. RTGs

( $34^{\circ}$ Between Neighboring RTGs, 18 Heat Source Modules per RTG)

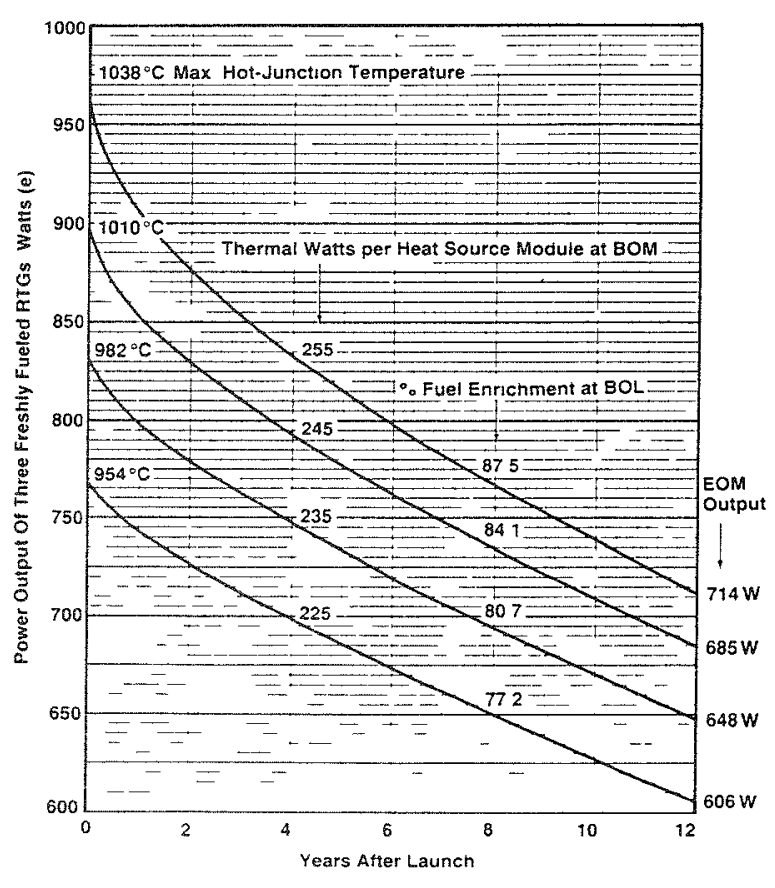

ACKNOWLEDGMENT

This work was supported by the U.S. Department of Energy's Office of Special Applications. The author is happy to acknowledge the contributions of C.T. Or and $H$. Noravian, who applied the equations presented here to generate the illustrative results shown in Figures 4 and 6

\section{REFERENCES}

[1] A Schock, H Noravian, and C. Or (1990) "Coupled Thermal and Electrical Analysis of Obstructed RTGS," Proc. of the 25 th Intersociety Energy Conversion Engineering Conference, held in Reno, Nevada, August 1990.

[2] A Schock, H. Noravian, C. Or, and $K$. Sankarankandath (1991) "Design and Analysis of RTGs for CRAF and Cassini Missions," Trans. of the 8th Symposium on Space Nuclear Power Systems, held in Albuquerque, New Mexico, January 1991.

[3] V. Raag and K.P. McCarron (1973) "Mathematical Model and Computer Program for the Design and Analysis of Silicon-Germanium Air-Vac RTG Degradation," Memorandum \#15, Syncal Corporation, Sunnyvale, California, October 1973.

[4] A.L. Mowery (1975) "Preliminary Power Degradation Report," Interoffice Memorandum to J. Lombardo, U.S. Energy Research and Development Administration, Washington, D.C., May 1975. 